\title{
When Food Can Be Fatal: Recovery from Emaciation
}

\author{
Diane Winn, PHD \\ AVIan HaVEN \\ FREEDOM, MAINE
}

\section{INTRODUCTION}

With the approach of winter, we can expect to admit increasing numbers of animals that have not been able to find sufficient food. With starvation comes emaciation-the loss of body fat and, as starvation continues, lean body mass. Progressive emaciation is sometimes categorized by amount of weight loss. However, the degree of emaciation in a given animal cannot be determined on the basis of body weight alone, because what is 'normal' for a given species depends on factors such as geographical location, time of year, and the sex of the animal. Gross signs of emaciation include prominent ribs (mammals) or keel (birds), and wasting leg muscles (reptiles).

\section{PHYSIOLOGY OF EMACIATION}

A more accurate way of describing the process of emaciation is as a series of physiological stages (Robbins 1993). Consider first a well-nourished animal: energy is obtained from ingested protein, fat and carbohydrate. Fat and carbohydrate not needed at the moment is stored; insulin levels are high. This state can be described as metabolically constructive (or anabolic).

When no more food remains in the gastrointestinal tract, insulin levels begin to drop. In Phase I, the animal utilizes stored carbohydrate (glycogen) as its primary fuel. This first phase of fasting is relatively short, though body weight drops rapidly within it. Phase II, which is characterized by the mobilization of fat for energy, lasts longer (depending on how much fat has been stored) and has a slower but constant weight loss. When both glycogen and fat reserves are depleted, the protein of muscle and other tissues is utilized; weight loss in Phase III is rapid. In these metabolically destructive (or catabolic) states, the body 'burns itself' for energy. Animals that store very little

Diane Winn is the co-founder and co-director of Avian Haven, a nonprofit wild bird rehabilitation center in Freedom, ME.

fat may progress through these phases very rapidly, being able to survive no more than a few days without food (Robbins 1993). Small birds and mammals are especially vulnerable because their relatively large surface area means high energy requirements. For these animals, survival in the cold temperatures of winter can be a particular challenge when ice storms, crusted snow, or other factors make food even more difficult to obtain.

\section{REFEEDING: ISSUES AND COMPLICATIONS}

For many people, the first impulse on rescuing a starving animal is to give it food. But doing so may have fatal consequences. One reason is that the organs of the gastrointestinal tract may be weakened by catabolism, reducing their ability to digest and absorb food. Another reason to avoid immediately refeeding an emaciated animal involves electrolyte imbalances that result from starvation. Although whole-body stores of phosphate, potassium and magnesium are depleted, serum concentrations remain normal at the expense of intracellular levels (those in the body's cells). When food is reintroduced, insulin levels surge and there is a sudden demand for electrolytes within the body's cells. The shift of extracellular (or serum) phosphate, potassium and magnesium to the interior of the cells causes a profound drop in serum electrolytes.

These electrolyte depletions are a hallmark of Refeeding Syndrome (RFS), a potentially fatal condition that occurs when inappropriate nutrition is provided to emaciated animals. The earliest reports of RFS involved survivors of WWII concentration camps and the siege of Leningrad, some of whom died of cardiac failure after the reintroduction of food. Since that time, RFS has been recognized as a component of other conditions involving prolonged fasting, including anorexia nervosa. RFS in humans has been described in detail relatively recently (Brooks and Melnik 1995; Crook et al 2001; Marinella 2003; Solomon and Kirby 1990). Although little informa- 
tion has appeared in the veterinary or rehabilitation literature, Remillard et al 2000 contains a great deal of related material, and Tseng (2005) has given recent conference presentations on RFS. The important thing to know about RFS is that low levels of phosphate, potassium, and magnesium produce a great many adverse effects in a variety of organs (including heart, liver, and kidneys) and organ systems (including respiratory, neuromuscular, and gastrointestinal). In turn, these effects may produce cardiac (increased heart rate, congestive heart failure, cardiac arrest), respiratory (difficulty breathing), gastrointestinal (regurgitation, anorexia, constipation, diarrhea), neurological (ataxia, seizures, coma), and other clinical signs. Vitamin deficiencies (especially thiamine) and fluid imbalances compound the difficulties seen in RFS.

Treatment of RFS is extremely difficult; in humans, it would involve daily monitoring of various electrolytes, supplementing with each as appropriate. Even in human medicine, prevention is easier than treatment. Prevention of RFS begins with replenishing electrolytes and fluids before introducing food. When calories are given, the rule is 'start low and go slow' (Brooks and Melnik 1995) to avoid catapulting a catabolic patient into a potentially fatal anabolic state. Of course, even before fluids are given, an emaciated animal should be warmed, and kept in a thermoneutral environment (approaching body temperature) at least through the first few days of treatment.

Hydration. Oral rehydration fluids contain water and electrolytes; isotonic solutions such as Lactated Ringer's and Normosol-R ${ }^{\circledR}$ (Abbott Laboratories, Abbott Park, IL) may be safer in this context than those containing dextrose (which may precipitate rapid cellular fluid shifts). B-vitamins should be added to the fluids (Remillard et al 2000); one guideline is $1 \mathrm{ml}$ of injectable $\mathrm{B}$-complex per liter of fluids or 1 to $2 \mathrm{mg} / \mathrm{kg}$ SID dosed according to the amount of thiamine (Tseng 2005). In human medicine, electrolyte levels can usually be corrected over 12 to 24 hours, after which refeeding can begin (Crook et al 2001).

In determining the amount of fluids to administer, a good starting point is the daily fluid maintenance requirement: roughly $5 \%$ (or $50 \mathrm{ml} / \mathrm{kg}$ ) of body weight for mammals and birds (Miller and Wolf 2003) and 1 to 3\% for reptiles (Mitchell 2006). A greater amount would be needed to replenish fluids in a dehydrated animal (a standard three day protocol, giving fluid amounts based on 'normal' weight, can be found in sources such as Miller and Wolf 2003).
However, because of compromised kidney function, fluid overload is another complication of RFS. Therefore, although emaciated animals are usually also dehydrated, they may need a more gradual replenishment of fluids, and a more conservative approach would be based on current weight rather than presumed 'normal' weight.

Foods and Feeding. Refeeding should begin with simple foods that are easy to digest. In general, to avoid RFS, these foods should be relatively low in carbohydrate. Remillard et al (2000) also recommend beginning refeeding with a formula that has a macronutrient profile similar to what the liver is estimated to be using from body stores-e.g., primarily fat in the intermediate phase of emaciation. An additional consideration for a target caloric distribution of macronutrients is the animal's normal food consumption pattern (Donoghue 2006; Remillard et al 2000): the diets of carnivores and insectivores contain relatively high proportions of protein and fat, but foods eaten by herbivores and granivores are high in carbohydrate.

Liquid nutritional products for humans such as Ensure ${ }^{\circledR}$ (Abbott Laboratories, Abbott Park, IL) have a history of use in rehabilitation, but may not be ideal for all species. They typically contain significant amounts of sucrose, which is not part of most animals' diets and may be problematic for species lacking sucrase, the digestive enzyme needed to break it down. Vital ${ }^{\circledR}$ HN (Abbott Laboratories, Abbott Park, IL), a human elemental diet containing mostly non-sucrose carbohydrate, may be appropriate for some species in an early stage of emaciation. Veterinary liquid enteral products such as CliniCare ${ }^{\circledR}$ Canine/Feline Liquid Diet (Abbott Laboratories, Abbott Park, IL) and Formula $\mathrm{V}^{\circledR}$ Enteral Care ${ }^{\mathrm{TM}}$ (Pet Ag, Inc., Hampshire, IL) are lower in carbohydrate, with more moderate fat and protein (Formula $\mathrm{V}^{\circledR}$ comes in high-level and mid-level protein varieties). Semi-solid veterinary products include Eukanuba Maximum Calorie ${ }^{\mathrm{TM}}$ Canine \& Feline Canned Formula (The Iams Company, Dayton, $\mathrm{OH}$ ) and Hill's ${ }^{\circledR}$ Prescription Diet Canine Feline a/d ${ }^{\circledR}$ (Hill's Pet Nutrition, Inc., Topeka, KS). This list is not meant to be complete; other products are also available, and some are marketed for reptiles or birds. However, the caloric distribution of some bird-targeted products seems better suited for granivorous (perhaps, the typical companion bird) than for insectivorous or carnivorous species.

Tables showing daily caloric requirements for mammals, passerine and nonpasserine birds are given in many sources, including NWRA Quick Reference (Miller and Wolf 2003). Requirements for reptiles 
may be found in Donoghue (2006). The principles of calculating these requirements are also explained in both sources. In deciding how much to feed per day, remember the axiom of 'start low and go slow'. How low and slow to start and go depends on how emaciated the animal is; in severe cases, one might begin with as little as one-third of the basal requirement (or Metabolic Energy Coefficient-the number of calories needed at rest in a thermoneutral environment), ramping up gradually, over several days, to the full basal requirement and beyond. The calories per $\mathrm{ml}$ of the products mentioned above, along with their caloric distributions, are shown in Table 1.

Divide the desired number of kcal by the number

Table 1. Calories and caloric distribution (percent of total calories provided by each nutrient) of selected diets.

\begin{tabular}{|l|c|c|c|c|}
\hline Product & $\mathbf{k c a l} / \mathbf{m l}$ & \% protein & \% fat & \% carb. \\
\hline Vital $^{\circledR} \mathrm{HN}$ & 1 & 17 & 9 & 74 \\
\hline CliniCare $^{\circledR}$ & 1 & 30 & 45 & 25 \\
\hline${\text { Formula } \mathrm{V}^{\circledR} \mathrm{HLP}}^{\text {Formula }^{\circledR} \mathrm{MLP}}$ & 1.2 & 32 & 45 & 23 \\
\hline Hill's $^{\circledR} \mathrm{a} / \mathrm{d}^{\circledR}$ & 1.2 & 27 & 51 & 23 \\
\hline Eukanuba Max-Cal $^{\mathrm{TM}}$ & 1.3 & 34 & 53 & 13 \\
\hline
\end{tabular}

of $\mathrm{kcal} / \mathrm{ml}$ of product to obtain the number of $\mathrm{ml}$ of product to feed (note that $\mathrm{a} / \mathrm{d}^{\circledR}$ and $\mathrm{Max}-\mathrm{Ca}^{\mathrm{TM}}$ may need dilution with water for tube feeding). The water contained in or added to these products counts toward the animal's fluid requirement; multiply the product's percent water (given on the label) by the number of $\mathrm{ml}$ to be fed to obtain the amount of water included in the elemental diet. Administer additional fluids as needed to achieve the maintenance amount and help replenish losses.

In determining the amount per feeding, keep in mind that the maximum stomach capacity for most animals is roughly five percent of body weight; however, a better guideline for any debilitated animal would be 2 to $3 \%$. Frequent feeding of small amounts is preferable, unless contraindicated by the stress of handling.

There is likely to be little weight gain over the first few days, other than what would be accounted for by fluid replacement. The goal is to have no weight loss during this time period. When the animal is able to digest elementary foods and begins to gain weight, whole foods can gradually be introduced, pureed at first. The transition from the enteral diet to the natural diet might take place over several days to a week, and should be guided by changes in signs such as weight, feces production, strength, alertness, etc.

\section{CONCLUSION}

If this sounds complicated-it is! Dealing with emaciation requires reference information, mathematical calculations, specialized equipment and supplies, and an ability to tube feed (if necessary, practice on cadavers to improve proficiency). Emaciated animals can be extremely difficult to save, especially in the later stages, when significant body mass has been catabolized. Additionally, these animals often have other problems that may have resulted in starvation-an injury or an illness that compounds the difficulty of rehabilitation. Rehabilitators who are not prepared to implement an emaciation protocol should consider transferring any such animals they admit to someone who has more experience and who can teach them to handle these cases in the future.

[Author's Note: If considering $\mathrm{a} / \mathrm{d}^{\circledR}$, one concern is that its first ingredient, liver, contains high levels of purines. In primates, birds, and some reptiles, purines are metabolized into uric acid and excreted. However, starvation and dehydration can compromise the kidneys' ability to excrete uric acid (cf. Donoghue 2006; Mader 2006). Liver is also extremely high in vitamin $A$, an excess of which can be problematic for birds (cf. Koutsos et al 2003). Thus, $\mathrm{a} / \mathrm{d}^{\circledR}$ may not be a good refeeding diet for some species.]

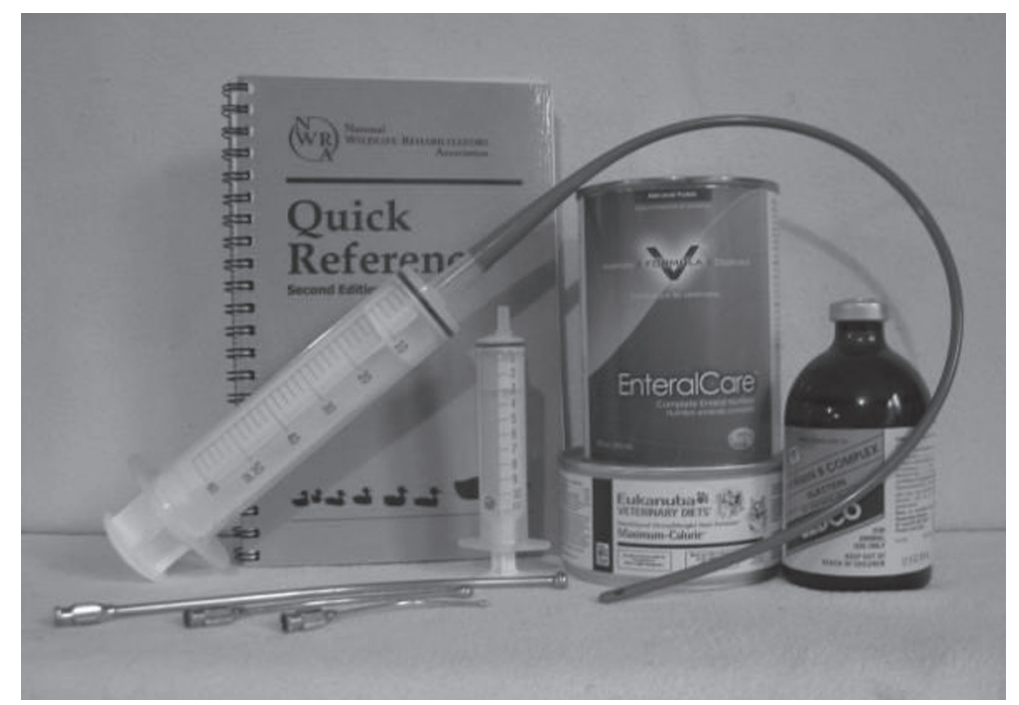

Supplies. 


\section{PRODUCT SOURCES}

NWRA Quick Reference can be ordered from NWRA (< http://www.nwrawildlife.org > through links to Marketplace, Publications).

Formula $V^{\circledR}$ Enteral Care, Vital ${ }^{\circledR}$ HN, gram scales, and gavage implements such as large-capacity syringes, stainless-steel and French catheter ('red rubber') feeding tubes are available at Squirrels and More (http://www.squirrelsandmore.com or 1-877-717-7748)

Electrolyte-containing fluids, CliniCare ${ }^{\circledR}$, Hill's $^{\circledR}$ $\mathrm{a} / \mathrm{d}^{\circledR}$, Eukanuba Max-Cal ${ }^{\mathrm{TM}}$, and other specialty diets can be ordered through your veterinarian.

\section{ACKNOWLEDGMENTS}

An earlier version of this article appeared in the Fall 2005 ReMaine Wild Newsletter. For answering questions, sharing protocols, and/or reviewing this manuscript, the author thanks Mark Finke, PhD; Erica Miller, DVM; Mark Mitchell, DVM; Mark Pokras, DVM; and especially Flo Tseng, DVM. Suggestions by anonymous reviewers for improving the manuscript are also appreciated.

\section{LITERATURE CITED}

Brooks, M., and G. Melnik. 1995. The refeeding syndrome: An approach to understanding its complications and preventing its occurrence. Pharmacotherapy. 15(6):713-726.

Crook, M., V. Hally, and J. Panteli. 2001. The importance of the refeeding syndrome. Nutrition. 17(7-8):632-637.

Donoghue, S. 2006. Nutrition. Pp. 251-298 in Reptile Medicine and Surgery (2nd edition) (D. Mader, ed.). Saunders Elsevier: St. Louis, MO.

Koutsos, E., L. Tell, L. Woods, and K. Klasing. 2003. Adult cockatiels (Nymphicus hollandicus) at maintenance are more sensitive to diets containing excess vitamin A than to vitamin A-deficient diets. Journal of Nutrition. 133(6):1898-1902.

Mader, D. 2006. Gout. Pp. 793-805 in Reptile Medicine and Surgery (2nd edition) (D. Mader, ed.). Saunders Elsevier: St. Louis, MO.

Marinella, M. 2003. The refeeding syndrome and hypophosphatemia. Nutrition Reviews. 61(9):320323.

Miller, E., and L. Wolf, eds. 2003. NWRA Quick Reference (2nd edition). National Wildlife Rehabilitators Association: St. Cloud, MN. Mitchell, M. 2006. Therapeutics. Pp. 631-664 in Reptile Medicine and Surgery (2nd edition) (D. Mader, ed.). Saunders Elsevier: St. Louis, MO.
Remillard, R., P. Armstrong, and D. Davenport. 2000. Assisted feeding in hospitalized patients: Enteral and parenteral nutrition. Pp. 351-399 in Small Animal Clinical Nutrition (M. Hand, C. Thatcher, R. Remillard, and P. Roudebush, eds.). Mark Morris Institute: Topeka, KS.

Robbins, C. T. 1993. Wildlife Feeding and Nutrition (2nd edition). Academic Press: San Diego, CA.

Solomon, S., and D. Kirby. 1990. The refeeding syndrome: A review. Journal of Parenteral and Enteral Nutrition. 14(1):90-97.

Tseng, F. 2005. Refeeding syndrome: How to avoid killing your patients with kindness. Presented at Wildlife Rehabilitators Association of Massachusetts (WRAM) Conference, Tufts University, February 2005. Nㅛㅛ

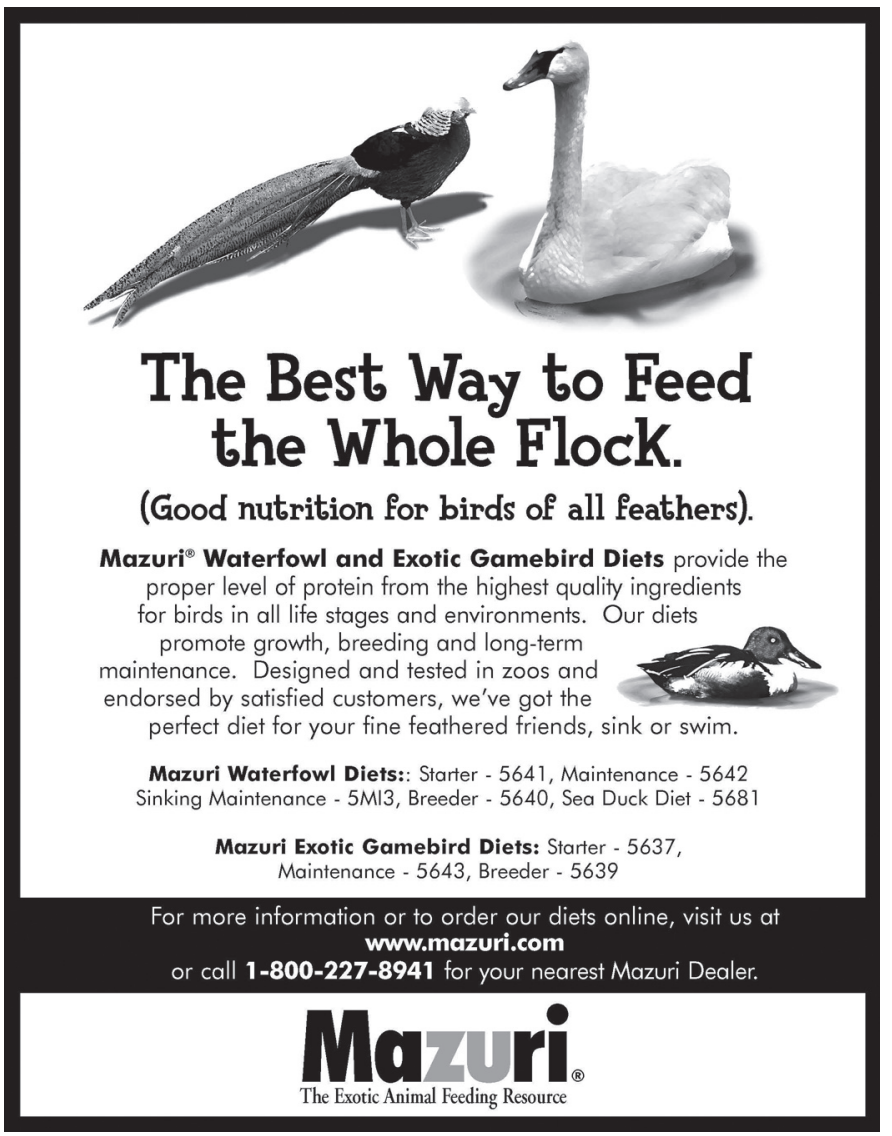

